\section{(6) OPEN ACCESS}

\title{
Changes in retinal venular oxygen saturation predict activity of proliferative diabetic retinopathy 3 months after panretinal photocoagulation
}

\author{
Thomas Lee Torp, ${ }^{1,2}$ Ryo Kawasaki, ${ }^{3}$ Tien Yin Wong, ${ }^{4}$ Tunde Peto, ${ }^{2,5}$ Jakob Grauslund ${ }^{1,2}$
}

\begin{abstract}
${ }^{1}$ Department of Ophthalmology, Odense University Hospital, Odense, Denmark

${ }^{2}$ Department of Clinical Research, University of Southern Denmark, Odense, Denmark ${ }^{3}$ Department of Public Health, Yamagata University Graduate School of Medical Science, Yamagata, Japan ${ }^{4}$ Singapore National Eye Centre, Duke NUS Medical School, National University of Singapore, Singapore, Singapore ${ }^{5}$ Department of Ophthalmology, Queen's University Belfast, Belfast, UK
\end{abstract}

\section{Correspondence to} Dr. Thomas Lee Torp, Department of Ophthalmology, Odense University Hospital, Sdr. Boulevard 29, Odense, Denmark; thomas.lee.torp@ rsyd.dk

Received 8 April 2017 Revised 12 June 2017 Accepted 16 June 2017 Published Online First 1 August 2017
Check for updates

To cite: Torp $T L$, Kawasaki R, Wong TY, et al. $\mathrm{Br} J$ Ophthalmol 2018;102:383-387.

\section{ABSTRACT}

Background/Aims Proliferative diabetic retinopathy (PDR) is a severe blinding condition. We investigated whether retinal metabolism, measured by retinal oximetry, may predict PDR activity after panretinal laser photocoagulation (PRP).

Methods We performed a prospective, interventional, clinical study of patients with treatment-naive PDR. Wide-field fluorescein angiography (OPTOS, Optomap) and global and focal retinal oximetry (Oxymap T1) were performed at baseline (BL), and 3 months (3M) after PRP. Angiographic findings were used to divide patients according to progression or non-progression of PDR after PRP. We evaluated differences in global and focal retinal oxygen saturation between patients with and without progression of PDR after PRP treatment.

Results We included 45 eyes of 37 patients (median age and duration of diabetes were 51.6 and 20 years). Eyes with progression of PDR developed a higher retinal venous oxygen saturation than eyes with nonprogression at 3M (global: $+5.9 \%(95 \% \mathrm{Cl}-1.5$ to 12.9), focal: $+5.4 \%$, (95\% Cl -4.1 to 14.8$))$. Likewise, progression of PDR was associated with a lower arteriovenular (AV) oxygen difference between BL and 3M (global: $-6.1 \%$, $(95 \% \mathrm{Cl}-13.4$ to -1.4$)$, focal: $-4.5 \%(95 \% \mathrm{Cl}-12.1$ to 3.2$))$. In a multiple logistic regression model, increment in global retinal venular oxygen saturation (OR 1.30 per 1\%-point increment, $p=0.017$ ) and decrement in AV oxygen saturation difference (OR 0.72 per $1 \%$-point increment, $p=0.016$ ) at $3 \mathrm{M}$ independently predicted progression of PDR. Conclusion Development of higher retinal venular and lower AV global oxygen saturation independently predicts progression of PDR despite standard PRP and might be a potential non-invasive marker of angiogenic disease activity.

\section{INTRODUCTION}

Diabetic retinopathy (DR) is a leading cause of blindness in the working-age population globally. ${ }^{1-3}$ Proliferative diabetic retinopathy (PDR) is an ischaemic end-stage complication characterised by new retinal vessels (proliferations) that often lead to vitreous haemorrhage and/or tractional retinal detachment. The Diabetic Retinopathy Study demonstrated that panretinal laser photocoagulation (PRP) reduces the risk of severe visual loss by $57 \%$ in patients with high-risk PDR. ${ }^{4}$ The purported mechanism of PRP has been debated for decades, but a general hypothesis is that energy delivered by the laser is absorbed by the retinal pigment epithelium and transformed to heat energy conducted to the neurosensory part of the retina, leading to thermic destruction of the affected retinal areas, which reduces the hypoxic load. ${ }^{4-6}$ While PRP is now considered standard of care for PDR, PRP is a global retinal treatment with little intra-individual variation. The inability to accurately predict progression of disease after PRP is partly because all patients are given the same amount of laser treatment. Some patients may have insufficient treatment while excessive treatment has been given to others. Hence, some might have disease progression with recurrent vitreous haemorrhage, and others may develop side effects like visual field loss, night blindness or diabetic macular oedema. ${ }^{5-7}$

The level of retinal metabolism may be an indicator of disease activity after PRP. This can be non-invasively measured by retinal oxygen saturation using a spectrophotometric retinal oximeter, ${ }^{8}$ and increasing levels of DR has been associated with increased retinal venular oxygen saturation. ${ }^{9-12}$ Hence, retinal oxygen saturation could act as an early indicator of PRP treatment response, and thereby be a valuable tool in the individualised patient management. In order to test if optimal levels of PRP can be assessed preoperatively, the main aim of this study was to investigate if retinal oximetry prior to PRP could be used as a non-invasive marker of postoperative PDR activity.

\section{MATERIALS AND METHODS}

We carried out a 3-month prospective, interventional study of 45 treatment-naive eyes with PDR referred to Odense University Hospital, Denmark between 1 August 2014 and 31 October 2015. We excluded patients with clinical significant macular oedema, previous PRP in the study eye, treatment-demanding cataract, age $<18$ years or pregnancy.

At baseline (BL), all patients provided a full medical history (including type of diabetes) and underwent a standard ophthalmic examination including slit lamp examination performed in mydriasis with tropicamide $10 \mathrm{mg} / \mathrm{mL}$ (Mydriacyl) and Phenylephedrine 10\% (Metaoxedrin), optical coherence tomography (OCT) by 3D OCT-2000 Spectral domain OCT (Topcon, Tokyo, Japan), and widefield fluorescein angiography (Optomap; Optos, Dunfermline, Scotland, UK). All examinations were done by trained personnel.

BL examinations included blood pressure (BP) on the upper arm using BP measuring equipment 


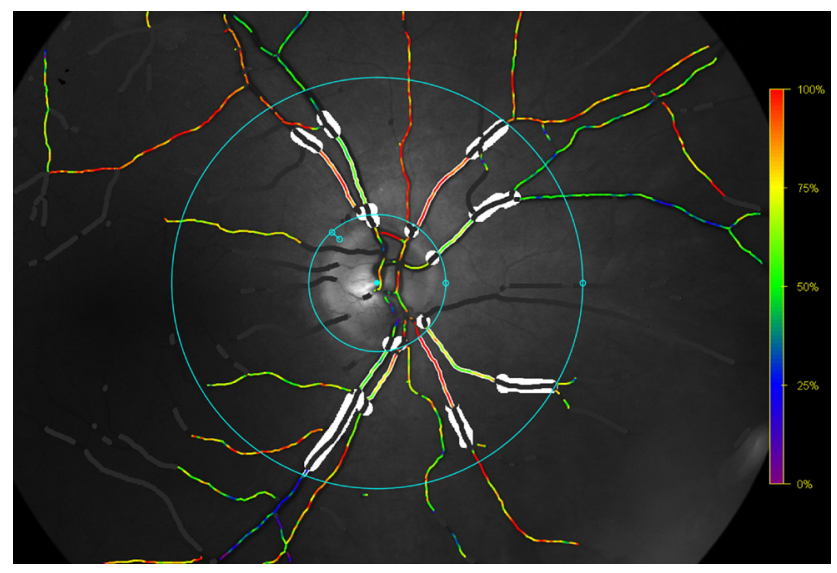

Figure 1 Retinal oxygen saturation. Oxymap T1 image showing traced vessels with overlaying colour map indicating oxygen saturation by colour on right eye. The oxygen saturation is measured in traced vessels, seen as white lines on either side of the vessels, between the blue rings. Retinal vessels covered in white areas are excluded vessel segments.

(Omron 705CP, Hoofdrop, The Netherlands), haemoglobin $\mathrm{A}_{1 \mathrm{c}}$ (HbA1c) and retinal oximetry (Oxymap model T1, Oxymap, software V.2.4.2, Reykjavik, Iceland).

Patients then received PRP in two sessions, 1 week apart by a navigated laser system (Navilas, OD-OS GmbH, Berlin, Germany). The patients were given a local anaesthetic (oxybuprocaine hydrochloride $0.4 \%$ ), and a Navilas $34 \mathrm{~mm}$ or $38 \mathrm{~mm}$ contact lens was used. All treatments were given by certified personnel (TLT and JG), and treatment specifications were automatically provided by the laser system after each session.

Follow-up was performed 3 months (3M) after PRP. Progression of PDR were defined as formation of new retinal proliferations, increased area of one or more proliferations or increased area of fluorescein leakage on angiography, as defined by clinical guidelines. Eyes with progression received supplementary PRP as needed, after follow-up examinations had been performed.

\section{Retinal oxygen saturation}

Oxymap T1 was used to assess the retinal oxygen saturation (figure 1). The equipment and technique are described elsewhere. ${ }^{13}$ All gradings were masked according to the PDR activity at follow-up and done by a single trained grader (TLT) in accordance with a prespecified grading protocol. Optic disc centred images were used for grading. Two rings were semi-automatically placed around the optic disc. The inner ring was manually placed between 20 and 30 pixels from the edge of the optic disc to avoid erroneous measurements due to reflection from the optic disc and the surrounding nerve fibre layer. The outer ring was automatically placed at three times the diameter of the inner ring. The oximetry measurements were done between the inner and outer ring. One larger arteriole and venule in each quadrant were automatically traced at a length of 50-200 pixels. If the length of the vessel was less than 50 pixels proximal to the first branching point, the first branch vessel was chosen for tracing. Measurements at follow-up were done on the same vessel segment. Two methods were used: Global measurements were defined as the mean retinal arteriolar and venular oxygen saturation of all four quadrants, and focal measurements were calculated as the retinal arteriolar and venular oxygen saturation of the affected retinal quadrant in patients with only one neovascularisation elsewhere. In addition to the arteriolar and venular measurements, the arteriovenular (AV) difference was included in the analysis.

\section{Approvals}

The study was approved by the Regional Scientific Ethics Committee (ID S-20140046), The Danish Data Protection Agency (ID 14/16546), registered at Clinical Trials on the fourth of June 2014 (ID NCT02157350), and performed in accordance with the criteria of the Helsinki II Declaration and good clinical practice. All patients gave informed consent before inclusion in the study. Furthermore, we attest that we have obtained appropriate permissions and paid any required fees for use of copyright-protected materials.

\section{Statistical analyses}

All statistical calculations were performed using STATA V.14.1 (StataCorp), and p values under 0.05 were considered statistically significant. At BL, categorical data are presented as percentage and continuous data as median with interquartile range (9IQR. Differences between patients with progression and non-progression of PDR were compared by the Mann-Whitney $U$ test for continuous data and $\chi^{2}$ for categorical data. Multiple logistic regression was performed with retinal oximetry measurements as predictors for progression of PDR (with adjustments for sex, age, duration of diabetes, HbA1c, systolic and diastolic BP and retinal laser energy). A standard power calculation on the arteriolar and venular oxygen saturation stated the inclusion of minimum 27 patients in the study (arteriolar: $\alpha$ 5\%, $\beta 20 \%$, mean 92.2, difference 2 , spread 3.7, $\mathrm{n}=27$, venular: $\alpha 5 \%, \beta$ $20 \%$, mean 55.6, difference 3.5 , spread $6.3, \mathrm{n}=26$ ).

\section{RESULTS}

A total of 45 eyes (37 patients) were examined. Nineteen patients had type 1 and 18 had type two diabetes. At BL, there was no significant difference in the retinal oxygen saturation between patients with type 1 and type two diabetes (arteriolar 96.1\% vs $97.0 \%, \mathrm{p}=0.79$ venular $64.4 \%$ vs $67.9 \%, \mathrm{p}=0.17)$, and therefore the data was merged.

The age and duration of diabetes (median and IQR) was $51.6 \pm 15$ and $20 \pm 11$ years, and $64 \%$ were male. HbA1c was $7.9 \% \pm 1.5 \%(63 \pm 16 \mathrm{mmol} / \mathrm{mol})$, and the median and IQR $\mathrm{BP}$ was $153 \pm 37 / 83.5 \pm 21 \mathrm{~mm} \mathrm{Hg}$. Retinal arteriolar and venular oxygen saturation was $96.3 \% \pm 7.2 \%$ and $67.2 \% \pm 13.6 \%$, respectively.

At baseline in global as well as focal analysis, patients with and without progression of PDR did not differ according to age, duration of diabetes, HbA1c, BP, total laser energy delivered, retinal oximetry image quality or retinal oxygen saturation (arteriolar, venular and arteriovenular difference) (table 1).

Between BL and $3 \mathrm{M}$, three eyes were excluded due to vitrectomy $(n=2)$ or death $(n=1)$, leaving a total of 42 eyes for global measurements and 24 for focal measurements. At $3 \mathrm{M}$ for global measurements, 12 eyes had progression of PDR as opposed to 30 eyes that did not progress. Correspondingly, in focal measurements, progression and non-progression of PDR was found for seven and 17 eyes, respectively.

\section{Retinal arteriolar oxygen saturation}

In global as well as focal measurements, there was no difference in retinal arteriolar oxygen saturation between the two groups, neither at $\mathrm{BL}, 3 \mathrm{M}$ or in the differences between $\mathrm{BL}$ and $3 \mathrm{M}$ (figure 2, figure 3, figure 4, figure 5 and table 2). 
Table 1 Baseline characteristics according to activity of proliferative diabetic retinopathy at follow-up month 3

\begin{tabular}{|c|c|c|c|c|c|c|}
\hline \multirow{2}{*}{$\begin{array}{l}\text { Baseline } \\
\text { characteristics }\end{array}$} & \multicolumn{3}{|c|}{ Global (median \pm IQR) } & \multicolumn{3}{|c|}{ Focal (median $\pm I Q R$ ) } \\
\hline & PDR progression & PDR non-progression & $p$ value & PDR progression & PDR non-progression & p value \\
\hline Eyes, $n$ & 12 & 30 & & 7 & 17 & \\
\hline Sex (men/women), $\mathrm{n}$ & $6 / 6$ & $22 / 8$ & 0.15 & $3 / 4$ & $14 / 3$ & 0.05 \\
\hline Age, years & $51.3 \pm 18.0$ & $53.8 \pm 26.0$ & 0.47 & $51.1 \pm 31.7$ & $42.3 \pm 21.8$ & 0.97 \\
\hline Diabetes duration, years & $15.5 \pm 22.5$ & $20 \pm 12.0$ & 0.28 & $12 \pm 8.0$ & $20 \pm 12.0$ & 0.07 \\
\hline $\mathrm{HbA} 1 \mathrm{c}, \%$ & $8.2 \pm 2.4$ & $7.9 \pm 1.5$ & 0.96 & $8.4 \pm 2.8$ & $7.8 \pm 1.2$ & 0.43 \\
\hline $\mathrm{HbA} 1 \mathrm{c}, \mathrm{mmol} / \mathrm{mol}$ & $66.5 \pm 26.5$ & $63 \pm 16.0$ & 0.96 & $68 \pm 31.0$ & $62 \pm 13.0$ & 0.43 \\
\hline $\begin{array}{l}\text { Systolic blood pressure, } \\
\mathrm{mm} \mathrm{Hg}\end{array}$ & $159.5 \pm 29.5$ & $153 \pm 39.0$ & 0.99 & $146 \pm 37.0$ & $140 \pm 29.0$ & 0.97 \\
\hline $\begin{array}{l}\text { Diastolic blood pressure, } \\
\mathrm{mm} \mathrm{Hg}\end{array}$ & $77.5 \pm 28.0$ & $83.5 \pm 12.0$ & 0.44 & $83 \pm 28.0$ & $82 \pm 14.0$ & 0.82 \\
\hline $\begin{array}{l}\text { Amount of laser energy, } \\
\text { Joule }\end{array}$ & $13.8 \pm 5.0$ & $13.2 \pm 4.6$ & 0.56 & $13.6 \pm 6.7$ & $11.5 \pm 4.1$ & 0.68 \\
\hline $\begin{array}{l}\text { Oximetry image quality, } \\
0-10\end{array}$ & $8.4 \pm 1.2$ & $8.0 \pm 1.0$ & 0.51 & $8.3 \pm 1.2$ & $8.1 \pm 0.9$ & 0.85 \\
\hline $\begin{array}{l}\text { Arteriolar oxygen } \\
\text { saturation, } \%\end{array}$ & $96.1 \pm 7.8$ & $95.3 \pm 7.8$ & 0.43 & $93.6 \pm 8.1$ & $97.6 \pm 9.0$ & 0.32 \\
\hline $\begin{array}{l}\text { Venular oxygen } \\
\text { saturation, \% }\end{array}$ & $65.2 \pm 16.3$ & $67.2 \pm 13.4$ & 0.96 & $62.0 \pm 18.6$ & $68.2 \pm 14.2$ & 0.59 \\
\hline $\begin{array}{l}\text { Arteriovenular oxygen } \\
\text { difference saturation, } \%\end{array}$ & $31.9 \pm 14.1$ & $30.2 \pm 11.1$ & 0.58 & $28.5 \pm 24.0$ & $34.6 \pm 20.6$ & 0.36 \\
\hline
\end{tabular}

Baseline characteristics in global and focal measurements according to activity of proliferative diabetic retinopathy (PDR) 3 months after panretinal laser photocoagulation.

Continuous data are presented as median with IQR. Differences between groups were compared with the Mann-Whitney $\mathrm{U}$ test for continuous data and $\chi^{2}$ test for categorical. Oximetry image quality ranged from $0=$ poor to $10=$ excellent.

$\mathrm{IQR}$, interquartile range; PDR, proliferative diabetic retinopathy.

\section{Retinal venular oxygen saturation}

There was a decrease in global retinal venular oxygen saturation between $\mathrm{BL}$ and $3 \mathrm{M}$ in patients without progression of PDR $(67.2 \%$ vs $63.8 \%, p=0.04$ ) (figure 2 ). Likewise, at $3 \mathrm{M}$, patients with progression of PDR had developed a higher difference in retinal venular oxygen saturation than patients without progression of PDR $(+4.1 \% \mathrm{vs}-1.8 \%, \mathrm{p}=0.02)$ (figure 4$)$. In a multiple logistic regression model, an increase in global retinal venular oxygen saturation between $\mathrm{BL}$ and $3 \mathrm{M}$ was independently associated with progression of PDR (OR 1.30 per 1\%-point increment, $95 \%$ CI 1.05 to $1.62, \mathrm{p}=0.02$ ) (table 2).

In the focal retinal venular oxygen measurements, there were no differences between $\mathrm{BL}$ and $3 \mathrm{M}$ between groups (figure 3),

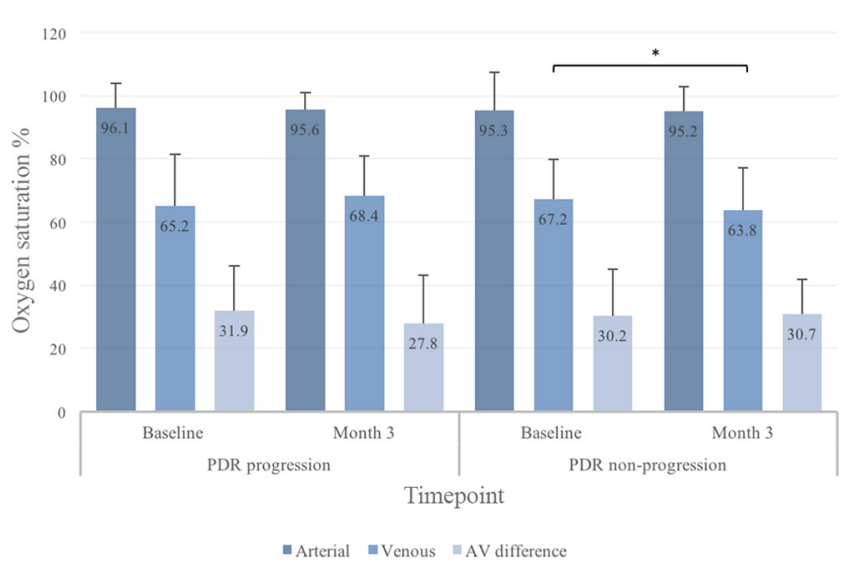

Figure 2 Global retinal oxygen saturation in eyes with progression or non-progression of proliferative diabetic retinopathy (PDR) before and after panretinal laser photocoagulation. All values are given as median with inter quartile range (IQR). AV, arteriovenular. *Statistically significant. but at $3 \mathrm{M}$, patients with progression of PDR had a higher increment from $\mathrm{BL}$ than patients without progression $(+3.9 \% \mathrm{vs}$ $-1.5 \%, \mathrm{p}=0.04$ ) (figure 5).

\section{Retinal AV oxygen difference}

In the global measurements, the retinal oxygen saturation AV difference between patients with progression and non-progression of PDR differed at $3 \mathrm{M}$ (progression $-4.1 \%$ vs non-progression $+2.0, p<0.01$ ) (figure 4 ). This was confirmed in the multiple logistic regression analysis (OR for progression of PDR 0.72 per $1.0 \%$-point increment in $\mathrm{AV}$ difference, $95 \% \mathrm{CI} 0.55$ to $0.94, \mathrm{p}=0.02)$ (table 2).

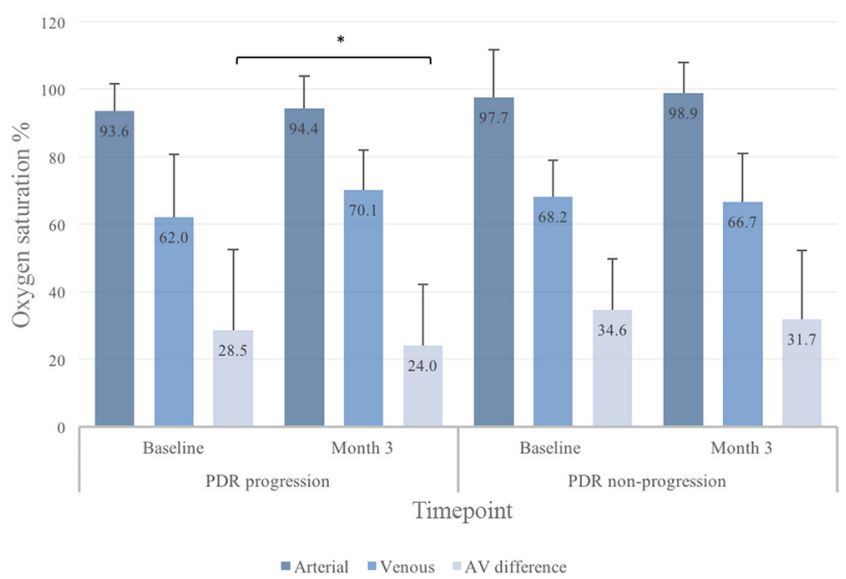

Figure 3 Focal retinal oxygen saturation in eyes with progression or non-progression of proliferative diabetic retinopathy (PDR) before and after panretinal laser photocoagulation. All values are given as median with interquartile range (IQR). AV, arteriovenular. ${ }^{*}$ Statistically significant. 


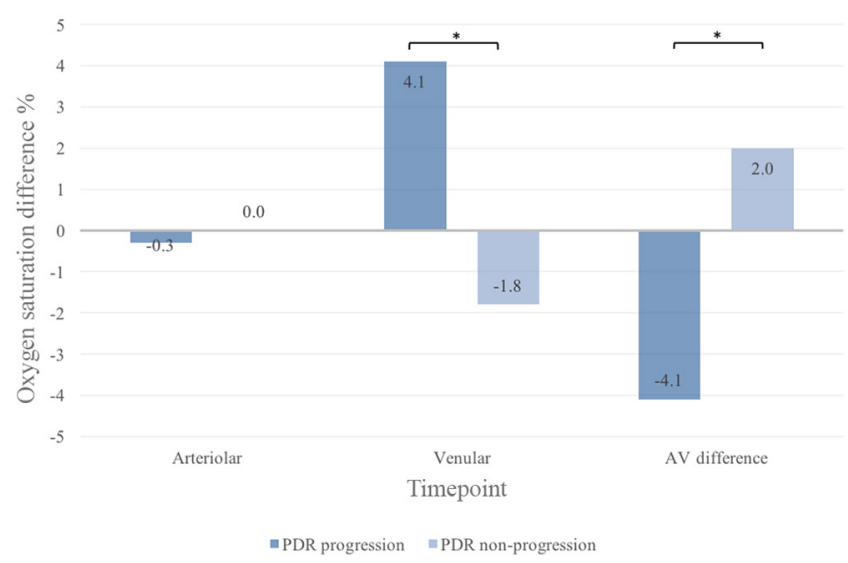

Figure 4 Difference in global retinal oxygen saturation from baseline to follow-up in eyes with proliferative diabetic retinopathy (PDR) according to disease activity at follow-up. All values are given as median. AV, arteriovenular. *Statistically significant.

In the focal analysis, a decrease in AV difference was seen between $\mathrm{BL}$ and $3 \mathrm{M}$ in patients with progression of PDR (28.5\% vs $24.0 \%, p=0.03$ ) (figure 3 ). There was also a different AV difference between patients with and without progression of PDR from BL to $3 \mathrm{M}(-3.1 \%$ vs $+1.4, \mathrm{p}<0.04)$ (figure 5$)$.

\section{DISCUSSION}

In this prospective study of patients with treatment-naive PDR, prelaser retinal oxygen saturation was not able to predict postoperative disease activity. However, the study also demonstrates postoperative changes in the retinal oxygen metabolism that were closely linked to disease activity 3 months after PRP. In fact, each $1 \%$ increase in retinal venular oxygen saturation was independently associated with a $30 \%$ higher risk of increased PDR activity despite laser treatment.

It has consistently been demonstrated that patients with DR have higher retinal venular oxygen saturation as compared with healthy controls. ${ }^{9} 111214$ Likewise, the retinal arteriolar and venular oxygen saturation increases with increasing levels of DR. ${ }^{10} 11$ The paradoxical difference between the high intravascular oxygen saturation and the ischaemic retinal tissue has been hypothesised to be caused by oxygen entrapment due to capillary closure, blood shunting around non-perfused retinal

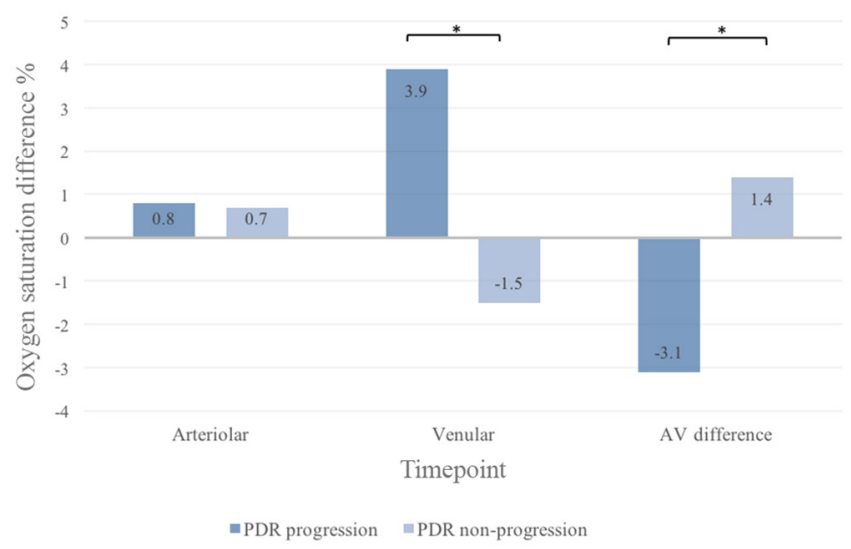

Figure 5 Difference in focal retinal oxygen saturation from baseline to follow-up in eyes with proliferative diabetic retinopathy (PDR) according to disease activity at follow-up. All values are given as median. AV, arteriovenular. *Statistically significant. areas, thickening of the capillary vessel walls, and greater affinity of haemoglobin for oxygen in patients with diabetes. ${ }^{11}$ An earlier study by Hammer et al demonstrated a trend towards lower retinal venular oxygen saturation in patients with PDR previously treated with PRP as compared with treatment-naive patients. ${ }^{12}$ In the present study, we demonstrated that this finding was only present in patients who were stabilised after PRP. This identifies retinal oxygen saturation as a non-invasive marker of disease activity, and might be of assistance to clinicians in the postoperative phase.

The retinal AV oxygen difference is considered as a pseudo-indicator of retinal oxygen consumption. ${ }^{11}$ In the present study, each 1\%-point increase in the retinal AV oxygen difference between $\mathrm{BL}$ and $3 \mathrm{M}$ independently reduced the risk of progression of PDR by $28 \%$, thereby indicating higher retinal oxygen use for those patients with a favourable treatment response. One explanation to the differences in retinal venular and AV oxygen saturation seen may be found in the hypoxic areas of the retina. These areas represent oxygen deficit tissue that react by increasing their retinal oxygen demand. This increase in oxygen demand causes the retinal oxygen saturation to increase, which unfortunately does not aid the hypoxic areas due to the overall poor condition of the capillaries. When successful PRP treatment is obtained, the hypoxic tissue is adequately decreased in area, thereby decreasing the retinal oxygen demand and lowering the venous and AV oxygen saturation.

We did not find the same differences in the arteriolar oxygen saturation. Earlier studies have shown a large difference in reported retinal arteriolar oxygen saturation as compared with the venules, ${ }^{111215}$ and this was the case despite a general higher retinal arteriolar oxygen saturation in patients with PDR. ${ }^{9} 1016$ This variation in reported retinal arteriolar oxygen saturation could be due to variance in laser treatment or oxygen saturation measurement protocols or equipment. Likewise, there could be a ceiling effect for arteriolar values that would not be present for venular measurements.

DR has traditionally been considered a global retinal ischaemic disease. However, some patients only have local lesions and may represent more focal phenotypes. In the present study, 24 patients only had a single proliferation elsewhere. For these patients, we were able to replicate the global oximetry findings with higher retinal venular oxygen saturation and lower AV differences at follow-up for patients with progression of PDR. Even though these findings are limited by a low number of patients in this subgroup, the focal findings should raise the question of a possible retained ischaemic overdrive in the retinal quadrant with the proliferation. Previous studies on healthy individuals have found regional differences in the retinal oxygen saturation, although the same difference has not been shown in patients with PDR. ${ }^{1718}$ These regional differences could explain why we did not find a difference in the oxygen saturation between the quadrant containing a proliferation and the oxygen saturation of the three adjacent quadrants (data not shown). Upcoming studies should address the possibility of a more focal treatment for patients with focal ischaemia.

Limitations of the study need to be considered. This includes a limited number of patients, which could affect the outcome of the study, although the power calculation was respected. An untreated observation group would have strengthened the study, but this was not possible due to ethical reasons. Furthermore, a trend towards a skewed distribution of women was seen in the focal measurements at $3 \mathrm{M}$. This may have confounded the interpretation of the focal results. Finally, retinal blood flow analyses were not included in the study. 
Table 2 Risk of progression of proliferative diabetic retinopathy after panretinal laser photocoagulation according to global and focal retinal oxygen saturation

\begin{tabular}{|c|c|c|c|c|c|c|c|}
\hline \multirow[b]{2}{*}{ Month 3} & \multirow[b]{2}{*}{ Increment } & \multicolumn{2}{|l|}{ Baseline } & \multicolumn{2}{|l|}{ Month 3} & \multicolumn{2}{|c|}{ Difference (month 3-baseline) } \\
\hline & & OR $(95 \% \mathrm{Cl})$ & $p$ value & OR $(95 \% \mathrm{Cl})$ & $p$ value & OR $(95 \% \mathrm{Cl})$ & $p$ value \\
\hline \multicolumn{8}{|l|}{ Global $(n=42)$} \\
\hline Arteriolar & $1.0 \%$ & $1.07(0.92$ to 1.24$)$ & 0.376 & $1.08(0.91$ to 1.27$)$ & 0.386 & $0.98(0.77$ to 1.26$)$ & 0.892 \\
\hline Venular & $1.0 \%$ & $0.99(0.89$ to 1.10$)$ & 0.885 & $1.12(0.98$ to 1.28$)$ & 0.087 & 1.30 (1.05 to 1.62$)$ & $0.017^{*}$ \\
\hline Arteriovenular & $1.0 \%$ & $1.05(0.94$ to 1.18$)$ & 0.390 & $0.95(0.85$ to 1.05$)$ & 0.320 & $0.72(0.55$ to 0.94$)$ & $0.016^{*}$ \\
\hline \multicolumn{8}{|l|}{ Focal $(n=24)$} \\
\hline Arteriolar & $1.0 \%$ & $0.86(0.65$ to 1.14$)$ & 0.285 & $0.98(0.83$ to 1.15$)$ & 0.768 & $1.30(0.81$ to 2.01$)$ & 0.274 \\
\hline Venular & $1.0 \%$ & 0.90 (0.75 to 1.09$)$ & 0.290 & 1.00 (0.86 to 1.16$)$ & 0.980 & $1.33(0.88$ to 1.99$)$ & 0.173 \\
\hline Arteriovenular & $1.0 \%$ & 1.02 (0.88 to 1.19$)$ & 0.756 & 0.99 (0.86 to 1.13$)$ & 0.835 & 0.89 (0.68 to 1.15$)$ & 0.360 \\
\hline
\end{tabular}

Multiple logistic regression model (adjusted for age, sex, duration of diabetes, $\mathrm{HbA1C}$, and amount of laser energy) indicating risk of progression of proliferative diabetic retinopathy 3 months after panretinal laser photocoagulation according to level of retinal oxygen saturation at baseline, follow-up and between baseline and follow-up. Risk indicated as odds ratio (OR) with $95 \%$ confidence interval (Cl). *Statistically significant.

In conclusion, the present study demonstrates that in patients with treatment-naive PDR, the retinal oxygen saturation cannot be used as a preoperative indicator of treatment outcome, but that the postoperative retinal oxygen metabolism closely reflects the disease activity and, hence, may be used to assess the need of adjunctive laser therapy. Upcoming studies should address if less invasive treatment of patients with focal PDR have the same efficacy but fewer side effects than standard-of-care PRP.

Acknowledgements We thank the Velux foundation and the Region of Southern Denmark for the financial support for this study. We also thank the Department of Ophthalmology, Odense University Hospital for providing the facilities and equipment necessary.

Contributors JG conceived the aims and overall design of the study. TLT acquired the data and did the writing of the different sections, tables and figures. JG and TLT did the literature search and statistical analyses. All authors were involved in the study design, data analyses, data interpretation and revision of the paper. The following authors had access to the full raw dataset: TLT and JG. The corresponding author had the final responsibility to submit for publication.

Funding The funders (the Velux Foundation and the Region of Southern Denmark) had no influence on the study design, data collection, analysis, interpretation of the data or writing of the report.

Competing interests None declared.

Patient consent Obtained.

Ethics approval The Regional Scientific Ethics Committee.

Provenance and peer review Not commissioned; externally peer reviewed.

Open Access This is an Open Access article distributed in accordance with the Creative Commons Attribution Non Commercial (CC BY-NC 4.0) license, which permits others to distribute, remix, adapt, build upon this work non-commercially, and license their derivative works on different terms, provided the original work is properly cited and the use is non-commercial. See: http://creativecommons.org/ licenses/by-nc/4.0/

(c) Article author(s) (or their employer(s) unless otherwise stated in the text of the article) 2018. All rights reserved. No commercial use is permitted unless otherwise expressly granted.

\section{REFERENCES}

1 Ting DS, Cheung GC, Wong TY. Diabetic retinopathy: global prevalence, Major risk factors, screening practices and public health challenges: a review. Clin Exp Ophthalmol 2016:44:260-77.
2 Klein R, Lee KE, Gangnon RE, et al. The 25-year incidence of visual impairment in type 1 diabetes mellitus the Wisconsin epidemiologic study of diabetic retinopathy. Ophthalmology 2010;117:63-70.

3 Liew G, Michaelides M, Bunce C. A comparison of the causes of blindness certifications in England and Wales in working age adults (16-64 years), 1999-2000 with 2009-2010. BMJ Open 2014;4:e004015.

4 Preliminary report on effects of photocoagulation therapy. the Diabetic Retinopathy Study Research Group. Am J Ophthalmol 1976;81:383-96.

5 Early photocoagulation for diabetic retinopathy. ETDRS report number 9. early treatment Diabetic Retinopathy Study Research Group. Ophthalmology 1991;98(5 Suppl):766-85.

6 Photocoagulation treatment of proliferative diabetic retinopathy. clinical application of Diabetic Retinopathy Study (DRS) findings, DRS Report Number 8. the Diabetic Retinopathy Study Research Group. Ophthalmology 1981;88:583-600.

7 Ferris FL, Podgor MJ, Davis MD. Macular edema in Diabetic Retinopathy Study patients. Diabetic Retinopathy Study Report Number 12. Ophthalmology 1987:94:754-60

8 Hardarson SH, Harris A, Karlsson RA, et al. Automatic retinal oximetry. Invest Ophthalmol Vis Sci 2006;47:5011-6.

9 Jørgensen CM, Hardarson SH, Bek T. The oxygen saturation in retinal vessels from diabetic patients depends on the severity and type of vision-threatening retinopathy. Acta Ophthalmol 2014;92:34-9.

10 Khoobehi B, Firn K, Thompson H, et al. Retinal arterial and venous oxygen saturation is altered in diabetic patients. Invest Ophthalmol Vis Sci 2013;54:7103-6.

11 Hardarson SH, Stefánsson E. Retinal oxygen saturation is altered in diabetic retinopathy. Br J Ophthalmol 2012;96:560-3.

12 Hammer M, Vilser W, Riemer T, et al. Diabetic patients with retinopathy show increased retinal venous oxygen saturation. Graefes Arch Clin Exp Ophthalmol 2009;247:1025-30.

13 Geirsdottir A, Palsson 0, Hardarson SH, et al. Retinal vessel oxygen saturation in healthy individuals. Invest Ophthalmol Vis Sci 2012;53:5433-42.

14 Rilvén S, Torp TL, Grauslund J. Retinal oximetry in patients with ischaemic retinal diseases. Acta Ophthalmol 2017:95.

15 Man RE, Sasongko MB, Xie J, et al. Associations of retinal oximetry in persons with diabetes. Clin Exp Ophthalmol 2015;43:124-31.

16 Jørgensen C, Bek T. Increasing oxygen saturation in larger retinal vessels after photocoagulation for diabetic retinopathy. Invest Ophthalmol Vis Sci 2014;55:5365-9

17 Jørgensen CM, Bek T. Lack of differences in the regional variation of oxygen saturation in larger retinal vessels in diabetic maculopathy and proliferative diabetic retinopathy. Br J Ophthalmol 2017;101.

18 Heitmar R, Safeen S. Regional differences in oxygen saturation in retinal arterioles and venules. Graefes Arch Clin Exp Ophthalmol 2012;250:1429-34. 